\title{
Effects of dialysis modality choice on the survival of end-stage renal disease patients in southern China: a retrospective cohort study
}

Zhiren He ${ }^{1,2+}$, Haijing Hou ${ }^{1,2+}$, Difei Zhang ${ }^{1,2^{*}}$, Yenan Mo ${ }^{2}$, La Zhang ${ }^{1,2}$, Guobin Su ${ }^{1,2}$, Junjie Lin ${ }^{1,2}$, Liming Lư ${ }^{3}$, Jingyao Huang ${ }^{2}$, Yewen $\mathrm{Gu}^{2}$, Ying Zhang ${ }^{4}$, Jingxia Lin ${ }^{1,2}$, Fengling Yuan ${ }^{1,2}$, Yu Peng ${ }^{1,2}$, Hui Liang ${ }^{1,2}$, Daixin Zhao ${ }^{1,2}$, Fuhua Lu ${ }^{1,2}$, Xusheng Liu ${ }^{1,2}$ and Lixin Wang ${ }^{1,2^{*}}$ (D)

\begin{abstract}
Background: The optimal choice of treatment, with hemodialysis (HD) or peritoneal dialysis (PD), for end-stage renal disease (ESRD) patients, is still controversial. Only a few studies comparing HD and PD have been conducted in China, which has the largest number of dialysis patients in the world.

Methods: A retrospective cohort study was conducted on ESRD patients who began renal replacement treatment from January 1, 2012 to December 31, 2017 in Guangdong Provincial Hospital of Chinese Medicine. Propensity scoring match was applied to balance the baseline conditions and multivariate Cox regression analysis to compare the mortality between HD and PD patients, and evaluated the correlation between mortality and various baseline characteristics.
\end{abstract}

Results: A total of $436 \mathrm{HD}$ patients and 501 PD patients were included in this study, and PD patients had better survival than HD patients, but the difference was not statistically significant. For younger ESRD patients ( $\leq 60$-yearold), the overall survival of PD was better than that of HD, but HD was associated with a lower risk of death in older patients (> 70-year-old). This difference was still significant after adjustment for a variety of confounding factors. Female gender, age at dialysis initiation, cardiovascular disease, cholesterol, and HD were risk factors of allcause mortality in the younger subgroup, while PD was risk factor in the older subgroup.

Conclusion: PD may be a better choice for younger ESRD patients, and HD for the older patients.

Keywords: Peritoneal dialysis, Hemodialysis, Age, End-stage renal disease, All-cause mortality

\footnotetext{
*Correspondence: zdf_doctor@163.com; wanglixin1210@163.com

'Zhiren He and Haijing Hou contributed equally to this work as first author.

'Department of Nephrology, The Second Affiliated Hospital of Guangzhou University of Chinese Medicine (Guangdong Provincial Hospital of Chinese Medicine), No. 111 Dade Road, Guangzhou 510120, Guangdong, China

Full list of author information is available at the end of the article
}

(C) The Author(s). 2020 Open Access This article is licensed under a Creative Commons Attribution 4.0 International License, which permits use, sharing, adaptation, distribution and reproduction in any medium or format, as long as you give appropriate credit to the original author(s) and the source, provide a link to the Creative Commons licence, and indicate if changes were made. The images or other third party material in this article are included in the article's Creative Commons licence, unless indicated otherwise in a credit line to the material. If material is not included in the article's Creative Commons licence and your intended use is not permitted by statutory regulation or exceeds the permitted use, you will need to obtain permission directly from the copyright holder. To view a copy of this licence, visit http://creativecommons.org/licenses/by/4.0/. The Creative Commons Public Domain Dedication waiver (http://creativecommons.org/publicdomain/zero/1.0/) applies to the data made available in this article, unless otherwise stated in a credit line to the data. 


\section{Background}

China has a large territory, a huge population, and insufficient medical resources. The number of endstage renal disease (ESRD) patients is continually rising. Hemodialysis (HD) is currently the main method of renal replacement therapy, and the number of peritoneal dialysis (PD) patients has increased rapidly [1]. The prevalence of $\mathrm{HD}$ and $\mathrm{PD}$ is estimated to be 402.18 and 39.95 per million individuals in China, and the corresponding number was approximately 553,000 HD patients and 55,000 PD patients in 2015 [2].

Whether HD or PD is a better choice for dialysis patients is still controversial [3]. Survival outcomes are major indicators for evaluating the efficacy and effectiveness of these two dialysis modalities. The impact of $\mathrm{HD}$ and $\mathrm{PD}$ on patient survival is conducted via randomized controlled trial (RCT), which is not commonly performed in dialysis patients because they developed a strong preference for a specific dialysis modality while being educated about the treatment. Only one RCT was stopped prematurely due to the low inclusion rate [4]. Several observational studies have compared PD and $\mathrm{HD}$ and attained conflicting results. Studies from Norway [5] and Denmark [6] have reported improved survival in PD patients, while some studies from Korea [7] and Singapore [8] have pointed to a survival benefit for HD patients. Nonetheless, there no evidence for a difference between both modalities in studies from Canada [9, 10], USA [11], and Taiwan [12]. Recently, a single-center retrospective study from Northern China showed a similar effect of HD and PD [13]. However, this study had limitations of small sample size and short duration of follow-up. Moreover, factors including climate condition, living habits, and economic resources vary across different regions in China, and might affect the survival outcomes of $\mathrm{HD}$ and $\mathrm{PD}$ patients. Therefore, the actual effect of HD and PD on survival is yet to be elucidated [3].

In order to compare the survival outcomes of patients using different dialysis modalities, a retrospective cohort study was conducted on ESRD patients who needed renal replacement treatment in Guangdong Provincial Hospital of Chinese Medicine in Southern China. Herein, we presented the results of the comparison of mortality between HD and PD patients and evaluated the correlation between mortality and various baseline covariates.

\section{Methods}

A retrospective cohort study was conducted from January 1, 2012 to December 31, 2017 in Guangdong Provincial Hospital of Chinese Medicine Nephrology
Department. The inclusion criterion was this period judged by the clinician during which long-term maintenance of renal replacement therapy was needed.

The outcome events were determined from previous follow-up records. The main outcome was all-cause mortality of the patient. The censor event included the patient switching to another dialysis mode or undergoing a kidney transplant or transfer to another dialysis center to continue treatment or reach the end of followup (December 31, 2018). The HD patients needed to return to the dialysis center for dialysis treatment 2-3 times a week; hence, the event could be recorded with a date. The majority of the PD patients were treated with continuous ambulatory peritoneal dialysis (CAPD) and needed to revisit every month for therapeutics. Therefore, follow-up visits were scheduled every 1-3 months, and the exact occurrences of the events were clarified by interviewing family members.

Baseline demographics, comorbid conditions, and laboratory test results were obtained by reviewing the electronic medical records. Demographic data included the date of birth, gender, the start of dialysis, the primary onset of kidney, height, and weight at the start of dialysis. The comorbidities were identified at the baseline by the International Classification of Diseases, 9th and 10th Revision (ICD-9 and ICD-10) codes, and the Charlson comorbidities index (CCI) was calculated based on Quan et al's method [14]. Cardiovascular diseases include asymptomatic myocardial ischemia (occult coronary heart disease), angina pectoris, myocardial infarction, ischemic heart failure (ischemic heart disease).

Laboratory indicators included hemoglobin, plasma albumin, serum creatinine, triglyceride, and cholesterol. These items were considered potential predictors or confounders.

Since this was a retrospective analysis, selection bias towards eligible patients causes an imbalance in the baseline status. Therefore, in the analysis, we used propensity score matching (PSM) to reduce the effect of selectivity bias. Furthermore, medical records were collected to deduce the statistical significance of the results. Based on the mortality rates reported in previous studies [8] in Singapore, the 5-year mortality difference between the two dialysis methods was $6.4 \%$ (PD: $13.4 \%$ vs. HD: $7.0 \%) ; \alpha=0.05$ and $\beta=0.10$. Using Pearson's chisquare test, the estimated sample sizes for a two-sample proportion is 872 cases, or 436 cases for each group.

Baseline characteristics and laboratory tests of HD patients were compared to those of PD patients. The normally distributed continuous variables were presented as mean \pm standard deviation (SD), and t-tests were used for comparison. Skewed data were presented as median and rank, and Mann-Whitney U test was used for comparison between groups. Missing data were filled in 
using a multiple imputation by chained equations algorithm by using R's MICE package. The filling method is "mean". The categorical variables were presented as percentages and analyzed by the chi-square test. The Kaplan-Meier survival curve was used to compare the overall survival between the initial dialysis modalities, and the significance of the difference was tested by the log-rank method.

Subgroup analyses were performed with respect to age. Previous studies suggested that patients undergoing HD and PD have different prognosis in different age subgroups. Therefore, we used Cox regression model to confirm the interactive effect of age and dialysis type. Further analysis of the interaction effects suggested that the age between 60 and 70 years may be the cutoff point for the difference in the prognosis of different dialysis methods. We found that in the subgroups of $\leq 60$-yearold, 60-70-year-old, and > 70-year-old, the type of dialysis had a significant effect on the prognosis.

Cox regression model was used to performed multivariate analysis (considering age, gender, primary kidney disease, and comorbidities). The covariates of multivariate regression were also determined based on clinical experience and previous studies, included sex, age at dialysis initiation, diabetes mellitus, cerebrovascular disease, congestive heart failure, chronic pulmonary disease, cardiovascular diseases, CCI, primary disease of renal failure (glomerulonephritis or polycystic kidney disease), body mass index, plasma albumin, cholesterol, and hemoglobin.

As a retrospective study, significant differences were detected in the baseline status, and hence, we used PSM to reduce these in different age groups by applying the MatchIt package in the R language for PSM at a ratio of 1:1. The matching method was nearest neighbor match. The characteristics used in PSM were the same as the variables in the multivariate Cox regression model.

Patients of different ages were grouped. Then single factor and multivariate Cox regression analysis were performed to determine the relative hazard ratio (HR) of the dialysis types, followed by PSM and multivariate Cox regression analysis to confirm the relative HR of the dialysis types.

All statistical tests were evaluated using a two-tailed 95\% confidence interval (CI), and $p<0.05$ was considered statistically significant. All statistical analyses were performed using the $\mathrm{R}$ language (version 3.6.0).

\section{Results}

Baseline characteristics of included patients

All patients, who started dialysis therapy between January 1, 2012 and December 31, 2017, were analyzed. A total of 1029 ESRD patients were included in this study, of whom 92 were excluded. Twenty-seven were excluded due to lack of baseline data, 3 were $<18$-year-old, 35 were due to a follow-up of $<3$ months, and 27 patients had maintained other dialysis methods for more than months before enrollment. Finally, there were 501 patients in the PD group and 436 in the HD group (Fig. 1).

Demographic and clinical characteristics are presented in Table 1. The mean age at dialysis start of pd. patients was younger than that of hemodialysis patients $(51.69 \pm$ 14.47 vs. $57.11 \pm 15.92$ years old, $p<0.01$ ). The proportion of females in patients receiving PD and HD was similar $(46.10 \%$ vs. $41.92 \%, p=0.22)$. The follow-up duration of the HD group was longer than that of the PD group (36.98 \pm 21.26 vs. $31.68 \pm 18.71$ months, $\mathrm{p}<0.01)$. This difference was observed across all age groups.

In terms of primary kidney disease, diabetic nephropathy and obstructive nephropathy were similar in the two groups. In terms of primary renal diseases, glomerulonephritis accounted for a high proportion in PD group. In the HD group, polycystic kidney occupies a higher proportion. At dialysis initiation, diabetes mellitus, congestive heart failure, and cerebrovascular disease were common among the HD patients, who also presented a high CCI value. In terms of lab tests, patients who started dialysis with HD had higher triglyceride, plasma albumin, and hemoglobin, and lower serum urea, serum creatinine, cholesterol, and prealbumin than those who started dialysis with PD. Similar differences were noted in the $\leq 60$-year-old group; however, it was not obvious in the $>70$-year-old group (Table 1).

\section{Comparison of survival rates between the PD and HD groups \\ Overall survival}

The 1-, 3-, and 5-year survival rates were 98.1, 86.7, and $73.4 \%$, respectively, in the PD group and 96.4, 83.0, and $71.9 \%$, respectively. in the HD group. The Kaplan-Meier survival analysis showed that the overall prognosis of the two dialysis methods varied, but not significantly $(p=$ 0.073, Fig. 2a).

\section{Subgroup analyses by age}

Cox regression analysis indicated that age was a risk factor for all-cause mortality in patients (Table 2). Previous studies from Asia have suggested that dialysis methods at different ages influence all-cause mortality. Thus, we performed a subgroup analysis based on age.

According to the interaction effect analysis, all patients were divided into three groups base on the age at dialysis initiation ( $\leq 60$-year-old, $60-70$-year-old, and $>70$ year-old). In the above age groups, the all-cause mortality rate ratio of HD to PD was 1.97 (95\% confidence interval (CI):1.13-3.49), 0.81 (95\% CI: 0.41-1.60), and 0.72 (95\% CI: $0.40-1.33$ ), respectively (Table 2 ). 
Patients received kidney replace treatment at our center

(2012.1.1 to 2017.12.31)

$\mathrm{N}=1029$

Exclusion ( $\mathrm{N}=92$ )

27 were lack of baseline data

3 were younger than 18 years old

35 were followed up less than 3 months

27 patients had maintained other dialysis

methods for more than 3 months before

enrollment

Enrollment

Total, $\mathrm{N}=937$

(HD, N=436; PD, N=501)

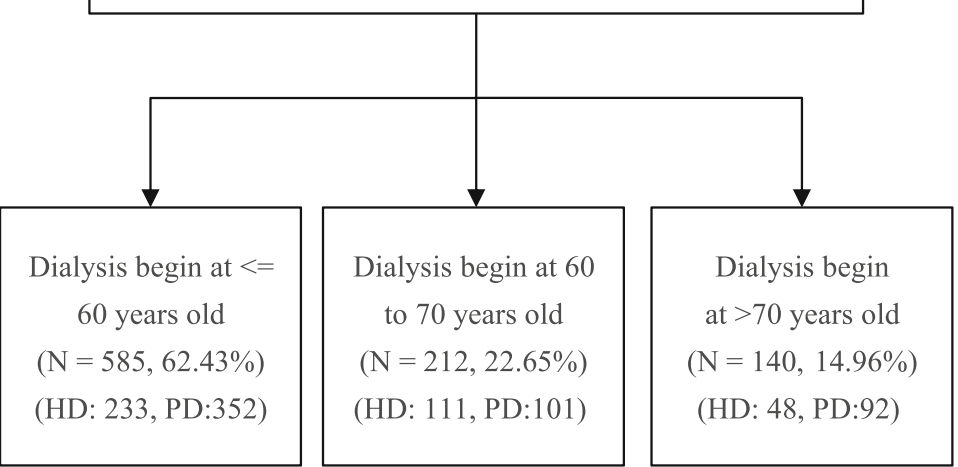

Fig. 1 Study schematic

In the $\leq 60$-year-old subgroup, the 1-, 3-, and 5-year survival rates of the PD group were 99.4, 93.2, and $85.2 \%$, while those of the HD group were 95.5, 87.0, and $81.8 \%$, respectively. The Kaplan-Meier survival analysis showed the survival rate of PD was significantly higher than that of HD ( $p=0.021$, Fig. 2b). In the $>70$-year-old subgroup, the 1-, 3-, and 5-year survival rates of PD group were $93.5,59.4$, and $26.0 \%$ and that of HD group were $95.5,73.2$, and $45.5 \%$, respectively, indicating that HD had a better prognosis in this age group, but did not reach statistical significance $(p=0.060$, Fig. $2 d)$. On the other hand, patients in 60-70-year-old group did not show a significant difference in survival rate between HD and PD (Fig. 2c).

\section{Factors associated with survival in dialysis patients}

Observing the Kaplan-Meier survival curve, the prognosis of different dialysis methods in the two subgroups $\leq 60$-year-old and $>70$-year-old might be the opposite. Therefore, we performed Cox regression model analysis on these two subgroups. Considering the difference in the baseline status between the two groups, we used Cox regression model analysis again after PSM to validate our results. After PSM, the sample sizes of $\leq 60$-year-old group changed from 233 in the HD group and 352 in the PD group to 226 in both groups, the sample sizes of $>70$-year-old group changed from 92 in the HD group and 48 in the PD group to 42 in both groups.

In the $\leq 60$-year-old subgroup, the univariate Cox regression model suggested that age at dialysis initiation, diabetes, CCI, cardiovascular disease, total cholesterol, and HD were risk factors for all-cause mortality. Cardiovascular disease, total cholesterol, and HD were still risk factors in the multivariable-adjusted model. The cholesterol and HD remained risk factors for multivariate Cox regression after using propensity scores to eliminate the differences in baseline characteristics. The HR of HD increased from 1.96 (95\% CI: $1.13-3.41)$ to 2.26 (95\% CI: 1.17-4.35). In the $>70$-year-old subgroup, the univariate Cox regression model did not find any risk factors for all-cause mortality, the multivariate Cox regression model suggested HD was a protective factor. After using 
Table 1 Baseline characteristics

\begin{tabular}{|c|c|c|c|c|c|c|c|c|c|}
\hline & \multicolumn{3}{|l|}{ All Patients } & \multicolumn{3}{|c|}{$\begin{array}{l}\text { Patients younger than } 60 \text { years } \\
\text { old }\end{array}$} & \multicolumn{3}{|c|}{ Patients older than 70 years old } \\
\hline & $\begin{array}{l}\text { PD Group } \\
(n=501)\end{array}$ & $\begin{array}{l}\text { HD Group } \\
(n=436)\end{array}$ & $p$ & $\begin{array}{l}\text { PD Group } \\
(n=352)\end{array}$ & $\begin{array}{l}\text { HD Group } \\
(n=233)\end{array}$ & $p$ & $\begin{array}{l}\text { PD Group } \\
(n=48)\end{array}$ & $\begin{array}{l}\text { HD Group } \\
(n=92)\end{array}$ & $p$ \\
\hline \multicolumn{10}{|l|}{ Demographic data } \\
\hline Female(n) & $210(41.92 \%)$ & $201(46.10 \%)$ & 0.22 & $151(42.90 \%)$ & $90(38.63 \%)$ & 0.35 & $19(39.58 \%)$ & $57(61.96 \%)$ & 0.02 \\
\hline Age at dialysis initiation (years) & $51.69 \pm 14.47$ & $57.11 \pm 15.92$ & 0.00 & $44.55 \pm 10.58$ & $45.26 \pm 11.29$ & 0.44 & $75.77 \pm 3.66$ & $77.49 \pm 4.49$ & 0.02 \\
\hline Body mass index & $22.60 \pm 3.91$ & $23.13 \pm 4.30$ & 0.05 & $22.34 \pm 3.55$ & $23.01 \pm 4.60$ & 0.06 & $22.75 \pm 3.07$ & $23.27 \pm 4.13$ & 0.40 \\
\hline Duration of follow up (months) & $31.68 \pm 18.71$ & $36.98 \pm 21.26$ & 0.00 & $32.80 \pm 19.44$ & $38.28 \pm 22.14$ & 0.00 & $26.16 \pm 15.48$ & $34.86 \pm 19.30$ & 0.00 \\
\hline \multicolumn{10}{|l|}{ Kidney primary disease } \\
\hline Diabetic nephropathy(n) & $138(27.54 \%)$ & $116(26.61 \%)$ & 0.80 & $81(23.01 \%)$ & $57(24.46 \%)$ & 0.76 & $21(43.75 \%)$ & $26(28.26 \%)$ & 0.10 \\
\hline Glomerulus nephritis(n) & 255 (50.90\%) & $136(31.19 \%)$ & 0.00 & $198(56.25 \%)$ & $100(42.92 \%)$ & 0.00 & $10(20.83 \%)$ & $15(16.30 \%)$ & 0.67 \\
\hline Polycystic kidney(n) & $3(0.60 \%)$ & 21 (4.82\%) & 0.00 & $0(0.00 \%)$ & $14(6.01 \%)$ & 0.00 & $1(2.08 \%)$ & $0(0.00 \%)$ & 0.74 \\
\hline Obstructive nephropathy(n) & 36 (7.19\%) & $21(4.82 \%)$ & 0.17 & $23(6.53 \%)$ & $9(3.86 \%)$ & 0.23 & $6(12.50 \%)$ & $3(3.26 \%)$ & 0.08 \\
\hline Other or unknow(n) & $69(13.77 \%)$ & $142(32.57 \%)$ & 0.00 & $50(14.20 \%)$ & $53(22.75 \%)$ & 0.01 & $10(20.83 \%)$ & $48(52.17 \%)$ & 0.00 \\
\hline \multicolumn{10}{|l|}{ Comorbidities } \\
\hline $\begin{array}{l}\text { Charlson Comorbidities Index } \\
\text { (CCI) }\end{array}$ & $5.07 \pm 2.25$ & $5.87 \pm 2.47$ & 0.00 & $4.11 \pm 1.69$ & $4.36 \pm 1.96$ & 0.10 & $8.50 \pm 1.49$ & $8.41 \pm 1.45$ & 0.74 \\
\hline Diabetes(n) & $164(32.73 \%)$ & 170 (38.99\%) & 0.05 & $92(26.14 \%)$ & $73(31.33 \%)$ & 0.20 & $26(54.17 \%)$ & $49(53.26 \%)$ & 0.94 \\
\hline Cardiovascular disease(n) & $62(12.38 \%)$ & $64(14.68 \%)$ & 0.35 & $24(6.82 \%)$ & $18(7.73 \%)$ & 0.80 & $16(33.33 \%)$ & $27(29.35 \%)$ & 0.77 \\
\hline Congestive heart failure(n) & $120(23.95 \%)$ & 155 (35.55\%) & 0.00 & $81(23.01 \%)$ & $63(27.04 \%)$ & 0.31 & $21(43.75 \%)$ & $46(50.00 \%)$ & 0.60 \\
\hline Cerebrovascular disease(n) & $54(10.78 \%)$ & $88(20.18 \%)$ & 0.00 & $16(4.55 \%)$ & $30(12.88 \%)$ & 0.00 & $15(31.25 \%)$ & $36(39.13 \%)$ & 0.46 \\
\hline Chronic pulmonary disease(n) & $30(5.99 \%)$ & $32(7.34 \%)$ & 0.48 & $16(4.55 \%)$ & $13(5.58 \%)$ & 0.71 & $8(16.67 \%)$ & $12(13.04 \%)$ & 0.74 \\
\hline \multicolumn{10}{|l|}{ Laboratory tests } \\
\hline Serum Urea $(\mathrm{mmol} / \mathrm{l})$ & $25.35 \pm 11.77$ & $22.26 \pm 11.31$ & 0.00 & $26.09 \pm 12.32$ & $22.65 \pm 11.56$ & 0.00 & $23.33 \pm 9.84$ & $19.12 \pm 9.04$ & 0.01 \\
\hline Serum creatinine (umol/l) & $\begin{array}{l}860.59 \pm \\
283.38\end{array}$ & $\begin{array}{l}745.81 \pm \\
319.76\end{array}$ & 0.00 & $\begin{array}{l}899.14 \pm \\
283.23\end{array}$ & $\begin{array}{l}804.08 \pm \\
332.03\end{array}$ & 0.00 & $\begin{array}{l}715.11 \pm \\
214.78\end{array}$ & $\begin{array}{l}582.44 \pm \\
268.22\end{array}$ & 0.00 \\
\hline Triglyceride (mmol/L) & $1.34 \pm 0.79$ & $1.43 \pm 1.04$ & 0.15 & $1.34 \pm 0.74$ & $1.40 \pm 0.80$ & 0.36 & $1.12 \pm 0.70$ & $1.35 \pm 0.79$ & 0.09 \\
\hline Cholesterol (mmol/L) & $4.54 \pm 1.35$ & $4.45 \pm 1.28$ & 0.31 & $4.59 \pm 1.40$ & $4.42 \pm 1.26$ & 0.15 & $4.37 \pm 1.35$ & $4.39 \pm 1.08$ & 0.93 \\
\hline Plasma albumin(g/L) & $34.87 \pm 5.13$ & $35.45 \pm 5.57$ & 0.10 & $34.99 \pm 5.24$ & $35.83 \pm 5.97$ & 0.08 & $34.09 \pm 4.90$ & $35.89 \pm 4.43$ & 0.03 \\
\hline Prealbumin(g/L) & $316.75 \pm 85.99$ & $278.91 \pm 91.84$ & 0.00 & $329.16 \pm 84.76$ & $295.88 \pm 97.73$ & 0.00 & $242.97 \pm 73.70$ & $252.48 \pm 76.24$ & 0.48 \\
\hline Hemoglobin(g/L) & $82.36 \pm 18.67$ & $86.66 \pm 20.85$ & 0.00 & $81.52 \pm 18.63$ & $87.30 \pm 21.23$ & 0.00 & $83.42 \pm 19.30$ & $87.00 \pm 21.52$ & 0.33 \\
\hline
\end{tabular}

PD Peritoneal dialysis, HD Hemodialysis

the PSM to eliminate the differences in baseline characteristics, HD remained a protective factor for multivariate Cox regression. The HR of HD decreased from 0.46 (95\% CI: $0.23-0.91)$ to 0.33 (95\% CI: $0.14-0.79)$ (Table 3).

\section{Short- and long-term survival}

We found that within the first 2 years after dialysis initiation, PD patients in the entire population had a tendency to survive better, but the benefit failed to reach statistical significance. In $<60$-year-old subgroup, PD patients had survival advantage reached statistical significance $(p=0.014$, Fig. $3 \mathrm{~b})$, but this advantage was not found in other subgroups (Fig. 3). After the second year of follow-up, no differences in survival were found between the two dialysis methods (Fig. 4).

\section{Discussion}

In this study, we compared HD and PD and found that the overall survival of PD was better than that of HD in the $\leq 60$-year-old group. This difference was still significant after the adjustment for a variety of confounding factors and PSM. The advantage of PD in young patients was first discovered in the current study. On the other hand, in the analysis of the univariate Cox regression model, the $>70$-year-old group did not prove the protective effect of HD. However, in the multivariate Cox regression model and the PSM multivariate Cox regression model, the protective effect of HD is statistically 
Comparison of survival rate between hemodialysis and peritonal dialysis by age subgroups

(A: all patients, B: $<=60$ years old group, C: $60-70$ years old group, D: $>70$ years old group)
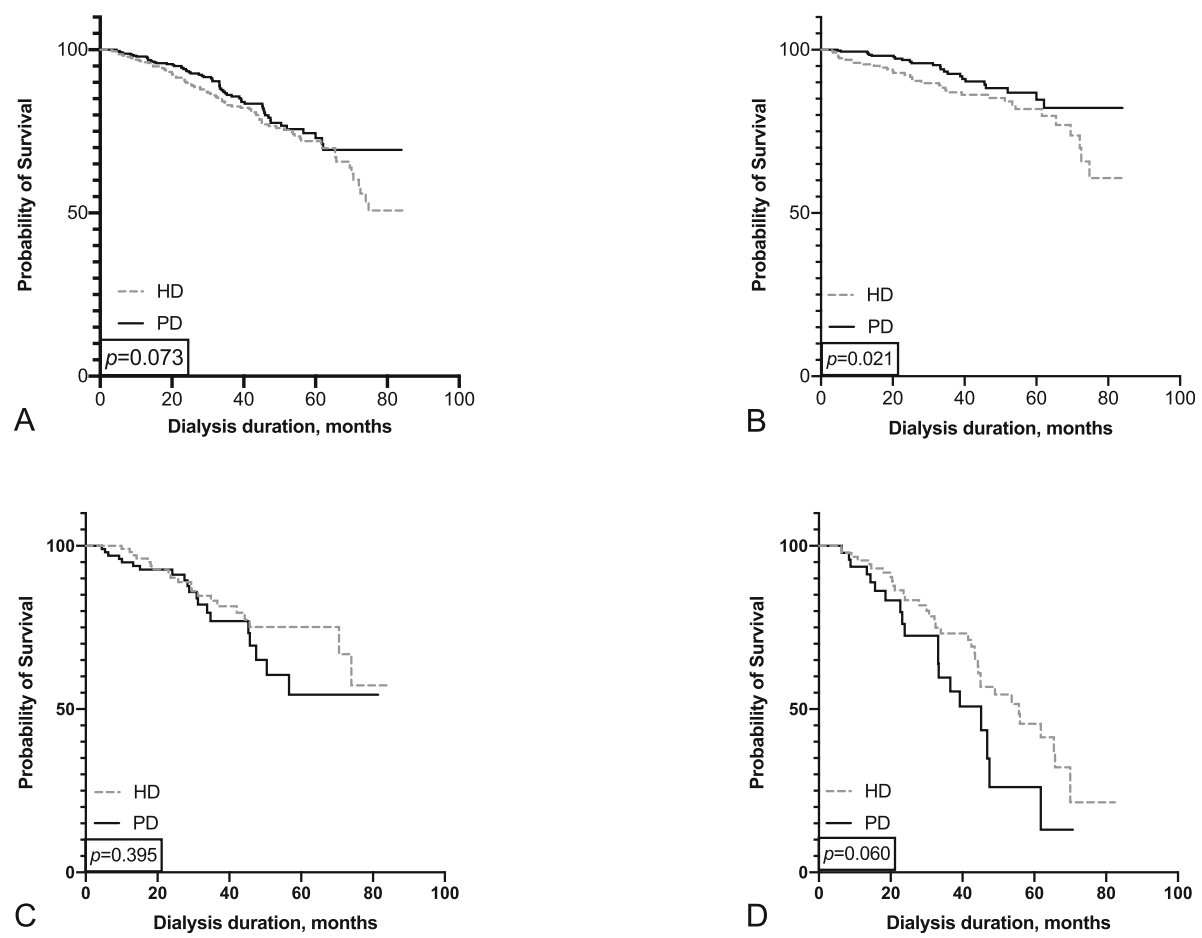

Fig. 2 Comparison of survival rate between hemodialysis and peritonal dialysis by age subgroups. (a: all patients, b: $<=60$ years old group, c: $60-$ 70 years old group, $\mathbf{d}:>70$ years old group)

significant. Our findings suggested that PD could be a better choice for younger patients $(\leq 60$-year-old), and HD could be a better choice for older patients $(>70$ year-old).

We compared $501 \mathrm{HD}$ patients and $436 \mathrm{PD}$ patients and found that PD patients had better survival rate than HD patients (1-, 3-, and 5-year survival rates were 98.1, 96.3, and $86.3 \%$ vs. $82.7,72.5$, and $71.1 \%$ ), albeit not significantly. The current result concurred with the observational study in Beijing, China [13]. A study with 871 ESRD patients in Singapore showed that patients who initiated dialysis with HD experienced better survival outcomes than those who initiated dialysis with PD [8]. This phenomenon might be related to the older age at dialysis start of PD patients (mean age 58.2-year-old for $\mathrm{HD}$ vs. 64.3-year-old for PD). Conversely, in this

Table 2 Cohort outcomes

\begin{tabular}{|c|c|c|c|c|}
\hline & All-cause mortality & Person-years & Mortality rate (dead/1000person-years) & Mortality rate ratio $(95 \%, \mathrm{Cl})$ \\
\hline \multicolumn{5}{|l|}{ All-Patients } \\
\hline $\mathrm{HD}(\mathrm{n}=436)$ & 90 & 1325.35 & 67.91 & $1.45(1.04-2.04)$ \\
\hline$P D(n=501)$ & 61 & 1304.52 & 46.76 & \\
\hline \multicolumn{5}{|c|}{ Age at dialysis initiation $<=60$} \\
\hline $\mathrm{HD}(\mathrm{n}=233)$ & 35 & 733.01 & 47.75 & $1.97(1.13-3.49)$ \\
\hline$P D(n=352)$ & 23 & 949.10 & 24.23 & \\
\hline \multicolumn{5}{|c|}{ Age at dialysis initiation 60-70 } \\
\hline $\mathrm{HD}(n=111)$ & 20 & 328.73 & 60.84 & $0.81(0.41-1.60)$ \\
\hline $\mathrm{PD}(n=101)$ & 19 & 252.23 & 75.33 & \\
\hline \multicolumn{5}{|c|}{ Age at dialysis initiation > 70} \\
\hline $\mathrm{HD}(\mathrm{n}=92)$ & 35 & 263.60 & 132.77 & $0.72(0.40-1.33)$ \\
\hline$P D(n=48)$ & 19 & 103.20 & 184.11 & \\
\hline
\end{tabular}


Table 3 Risk factors for mortality assessed by univariate and multivariate Cox regression model

\begin{tabular}{|c|c|c|c|c|c|c|}
\hline & \multicolumn{3}{|c|}{$\begin{array}{l}\text { Patients younger than } 60 \text { years old } \\
\text { PD }(n=352), \mathrm{HD}(n=233)\end{array}$} & \multicolumn{3}{|c|}{$\begin{array}{l}\text { Patients older than } 70 \text { years old } \\
\text { PD }(n=48), \operatorname{HD}(n=92)\end{array}$} \\
\hline & $\begin{array}{l}\text { Univariate } \\
\text { HR }(95 \% \mathrm{Cl})\end{array}$ & $\begin{array}{l}\text { Multivariate }^{\mathrm{a}} \\
\text { HR }(95 \% \mathrm{Cl})\end{array}$ & $\begin{array}{l}\text { PSM }^{b}-\text { Multivariate }^{a} \\
\text { PD and HD } \\
(n=226) \\
\text { HR }(95 \% \mathrm{Cl})\end{array}$ & $\begin{array}{l}\text { Univariate } \\
\text { HR }(95 \% \mathrm{CI})\end{array}$ & $\begin{array}{l}\text { Multivariate }^{\mathrm{a}} \\
\text { HR }(95 \% \mathrm{Cl})\end{array}$ & $\begin{array}{l}\text { PSM }^{\mathrm{b}}-\text { Multivariate } \\
\text { PD and HD } \\
(n=42) \\
\text { HR }(95 \% \mathrm{Cl})\end{array}$ \\
\hline Age at dialysis initiation (per 1 year) & $\begin{array}{l}1.03 \\
(1.00-1.06)\end{array}$ & $1.02(0.99-1.05)$ & $1.02(0.99-1.06)$ & $1.05(0.98-1.11)$ & $1.05(0.98-1.13)$ & $1.07(0.96-1.18)$ \\
\hline Sex (female vs male) & $\begin{array}{l}0.58 \\
(0.33-1.00)\end{array}$ & $0.53(0.29-0.95)$ & $0.58(0.30-1.13)$ & $0.83(0.49-1.42)$ & $0.90(0.49-1.63)$ & $1.14(0.47-2.75)$ \\
\hline Diabetes(n) & $\begin{array}{l}1.89 \\
(1.12-3.20)\end{array}$ & $1.14(0.62-2.11)$ & $1.26(0.46-3.44)$ & $1.00(0.58-1.70)$ & $1.13(0.61-2.09)$ & $1.77(0.61-5.17)$ \\
\hline Hypertension(n) & $\begin{array}{l}1.68 \\
(0.67-4.20)\end{array}$ & $1.33(0.50-3.52)$ & $1.44(0.43-4.86)$ & $1.18(0.16-8.56)$ & $1.52(0.19-12.24)$ & $0.95(0.10-8.92)$ \\
\hline Charlson Comorbidities Index (CCI) & $\begin{array}{l}1.23 \\
(1.07-1.41)\end{array}$ & $0.93(0.64-1.36)$ & $0.63(0.24-1.66)$ & $1.02(0.82-1.25)$ & $0.90(0.61-1.32)$ & $1.06(0.64-1.75)$ \\
\hline Congestive heart failure(n) & $\begin{array}{l}1.51 \\
(0.87-2.61)\end{array}$ & $1.11(0.61-2.00)$ & $1.22(0.56-2.65)$ & $1.08(0.63-1.85)$ & $0.99(0.52-1.85)$ & $1.35(0.49-3.71)$ \\
\hline Cerebrovascular disease(n) & $\begin{array}{l}1.72 \\
(0.78-3.80)\end{array}$ & $1.21(0.52-2.81)$ & $1.24(0.42-3.65)$ & $1.04(0.60-1.79)$ & $0.83(0.44-1.57)$ & $0.56(0.19-1.63)$ \\
\hline Chronic pulmonary disease(n) & $\begin{array}{l}1.42 \\
(0.51-3.93)\end{array}$ & $1.07(0.38-3.02)$ & $0.93(0.26-3.27)$ & $1.83(0.96-3.48)$ & $1.68(0.81-3.50)$ & $1.00(0.34-2.96)$ \\
\hline Cardiovascular disease(n) & $\begin{array}{l}2.87 \\
(1.41-5.86)\end{array}$ & $2.56(1.16-5.66)$ & $1.79(0.71-4.51)$ & $1.29(0.73-2.28)$ & $1.44(0.70-2.95)$ & $3.61(1.31-10.00)$ \\
\hline Body mass index (per 1 kg/m2) & $\begin{array}{l}1.04 \\
(0.98-1.11)\end{array}$ & $1.01(0.94-1.08)$ & $1.02(0.95-1.10)$ & $0.95(0.88-1.02)$ & $0.96(0.88-1.04)$ & $0.88(0.77-1.01)$ \\
\hline Hemoglobin (per $1 \mathrm{~g} / \mathrm{L}$ ) & $\begin{array}{l}1.01 \\
(0.99-1.02)\end{array}$ & $1.00(0.99-1.02)$ & $1.00(0.99-1.02)$ & $1.00(0.98-1.01)$ & $1.00(0.98-1.01)$ & $1.01(0.99-1.03)$ \\
\hline Plasma albumin (per $1 \mathrm{~g} / \mathrm{L}$ ) & $\begin{array}{l}0.97 \\
(0.93-1.02)\end{array}$ & $0.98(0.94-1.04)$ & $0.99(0.93-1.04)$ & $0.96(0.91-1.02)$ & $0.96(0.90-1.02)$ & $1.02(0.92-1.13)$ \\
\hline Triglyceride (per 1 mmol/L) & $\begin{array}{l}1.00 \\
(0.97-1.03)\end{array}$ & $1.03(0.98-1.08)$ & $1.20(0.78-1.84)$ & $0.98(0.95-1.02)$ & $1.00(0.94-1.05)$ & $1.41(0.63-3.12)$ \\
\hline Cholesterol (per $1 \mathrm{mmol} / \mathrm{L}$ ) & $\begin{array}{l}1.31 \\
(1.12-1.54)\end{array}$ & $1.37(1.16-1.63)$ & $1.27(1.02-1.58)$ & $1.04(0.79-1.36)$ & $1.07(0.79-1.46)$ & $1.13(0.73-1.75)$ \\
\hline Dialysis Methods (HD vs. PD) & $\begin{array}{l}1.85 \\
(1.09-3.14)\end{array}$ & $1.96(1.13-3.41)$ & $2.26(1.17-4.35)$ & $0.58(0.33-1.03)$ & $0.46(0.23-0.91)$ & $0.33(0.14-0.79)$ \\
\hline
\end{tabular}

PSM Propensity score match, HR Hazard ratio, 95\% Cl 95\% confidence interval, PD Peritoneal dialysis, HD Hemodialysis

a Variables adjusted in multivariate Cox model for both subgroups: sex, age at dialysis initiation, diabetes mellitus, cerebrovascular disease, congestive heart failure, chronic pulmonary disease, cardiovascular diseases, $\mathrm{CCl}$, primary disease of renal failure (glomerulonephritis or polycystic kidney disease), body mass index, plasma albumin, cholesterol, and hemoglobin.

${ }^{\mathrm{b}}$ The characteristics used in PSM included: sex, age at dialysis initiation, diabetes mellitus, cerebrovascular disease, congestive heart failure, chronic pulmonary disease, cardiovascular diseases, $\mathrm{CCl}$, primary disease of renal failure (glomerulonephritis or polycystic kidney disease), body mass index, plasma albumin, cholesterol, and hemoglobin.

cohort, patients with PD were younger (mean age 57.11year-old for HD vs. 51.69-year-old for PD). Thus, the age at which dialysis was started might be the key to patient survival. A 2008 study in Taiwan suggested that PD has a better prognosis in non-diabetic patients $<55$ year-old, while in other subgroups, the prognosis is similar [12]. Another study in South Korea demonstrated that the survival outcomes of these two dialysis modalities are similar for patients < 55-year-old, whereas HD is superior to PD for the elderly [7]. A study in Taiwan suggested that the overall survival time of PD patients has improved in recent years [12]. This phenomenon might be related to the continuous improvement of PD training methods and new dialysate applications [15]. Therefore, we speculate that the survival time of PD patients can be prolonged through better chronic disease management and peritoneal dialysis technology. Previous comparative studies in the United States [16], Australia and New Zealand [17] had found that peritoneal dialysis had a survival advantage during the first year or two of dialysis [18]. According to our data, we found that within the first 1-2 years after dialysis initiation, PD in the entire population had a tendency to survive better, but failed to reach statistical significance. In $<60$-yearold subgroup, PD had survival advantages, but the other subgroups did not find similar advantages. After the 
Comparison of survival between hemodialysis and peritoneal dialysis by age groups in first 2 years.

(A: all patients, B: <=60 years old group, C: $60-70$ years old group, D: $>70$ years old group)
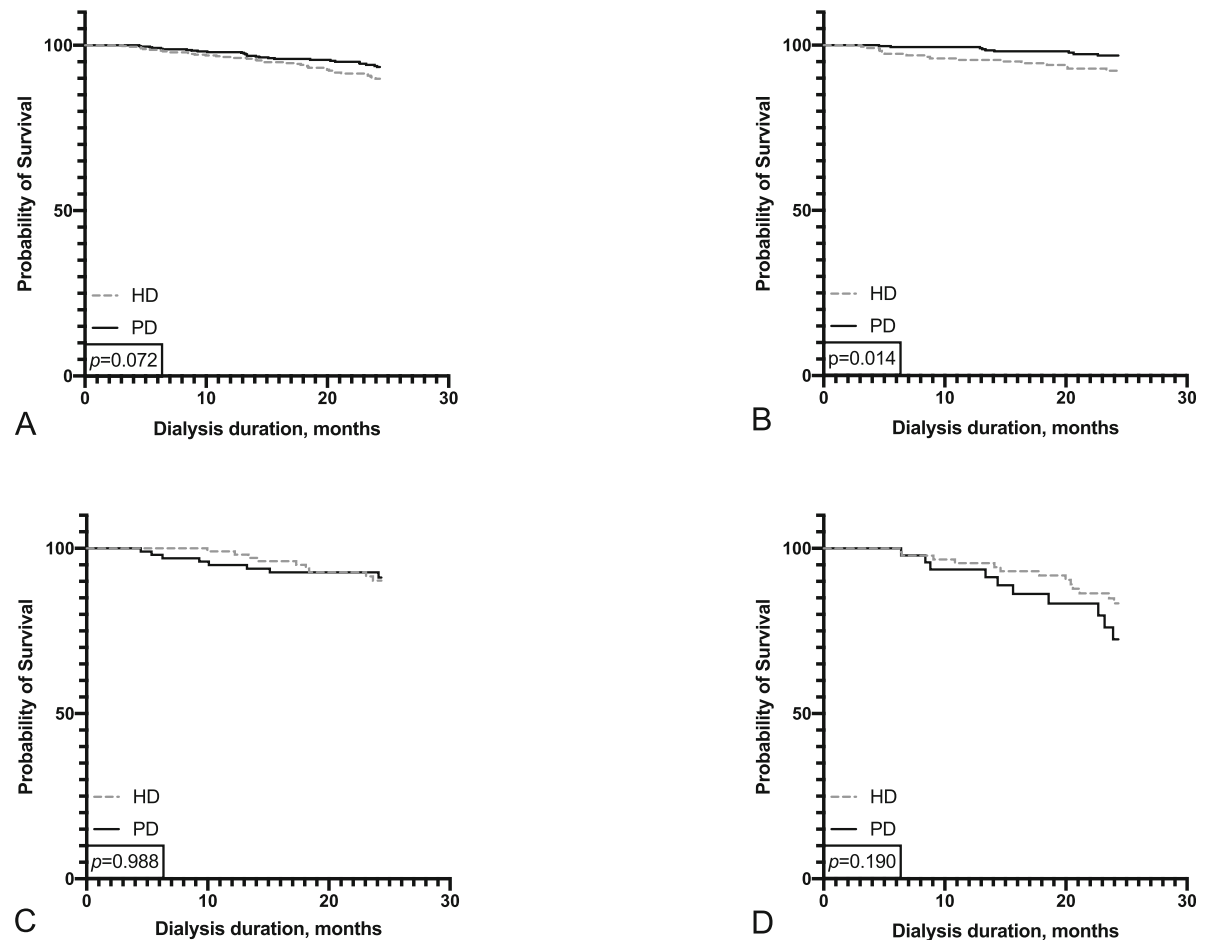

Fig. 3 Comparison of survival between hemodialysis and peritoneal dialysis by age groups in first 2 years. (a: all patients, $\mathbf{b}$ : $<=60$ years old group, c: $60-70$ years old group, $\mathbf{d}$ : $>70$ years old group)

second year of follow-up, no differences in survival were found between the two dialysis methods.

Regarding the risk factors associated with survival, age, diabetes [13, 19-21], serum albumin [19], BMI [13], and cardiovascular disease $[21,22]$ were not consistent with our results. Importantly, age is one of the major factors affecting survival in dialysis patients, which is consistent with our findings. Therefore, we performed subgroup analysis by age. Also, we found that the risk factors were not completely similar in different age subgroups $(\leq 60$ and $>70$-year-old). In the younger subgroup, CCI, cardiovascular disease, total cholesterol, and HD were risk factors of all-cause mortality. In the older subgroup, PD was risk factor.

The previous history of cardiovascular disease and diabetes mellitus was associated with left ventricular ejection fraction in PD patients [23]. Hypercholesterolemia was associated with increased mortality in HD patients without myocardial infarction/cardiovascular disease [24], while high pulmonary artery systolic pressure predicted the development of right ventricular dysfunction, which portends a poor prognosis [24]. The above three studies suggested that heart failure might be a main cause of mortality in young subgroups. PD might confer a survival advantage to young and healthier patients due to better preservation of residual renal function as compared to those undergoing HD [25]. The problem of ultrafiltration in the later period of PD could be solved by self-management and the use of icodextrin [25]. Therefore, the advantages of PD in younger subgroup might be due to the advantages of PD in the treatment of heart failure. Therefore, we performed a sensitivity analysis which includes patients with congestive heart failure only. The advantages of PD in young patients remained, which proved our speculation. However, the efficacy of HD and PD in patients with heart failure was still controversial. Further researches needed to be conducted.

CCI was originally used to estimate the 1-year mortality rate of hospitalized patients [26]. The condition of the population in this article is relatively mild and the followup time is longer, so CCI may not accurately reflect the impact of comorbidities on the prognosis. Hemmelgarn et al's study had shown that congestive heart failure, chronic pulmonary disease, and cardiovascular disease have a greater impact on the mortality of ESRD patients than other comorbidities [27]. Therefore, these comorbidities were added in to the model. However, due to the correlation between $\mathrm{CCI}$ and these comorbidities, the original model may have multicollinearity problems. For this 
Comparison of survival between hemodialysis and peritoneal dialysis by age groups from 2 years to end of follow up. (A: all patients, $B$ : $<=60$ years old group, C: $60-70$ years old group, D: $>70$ years old group)
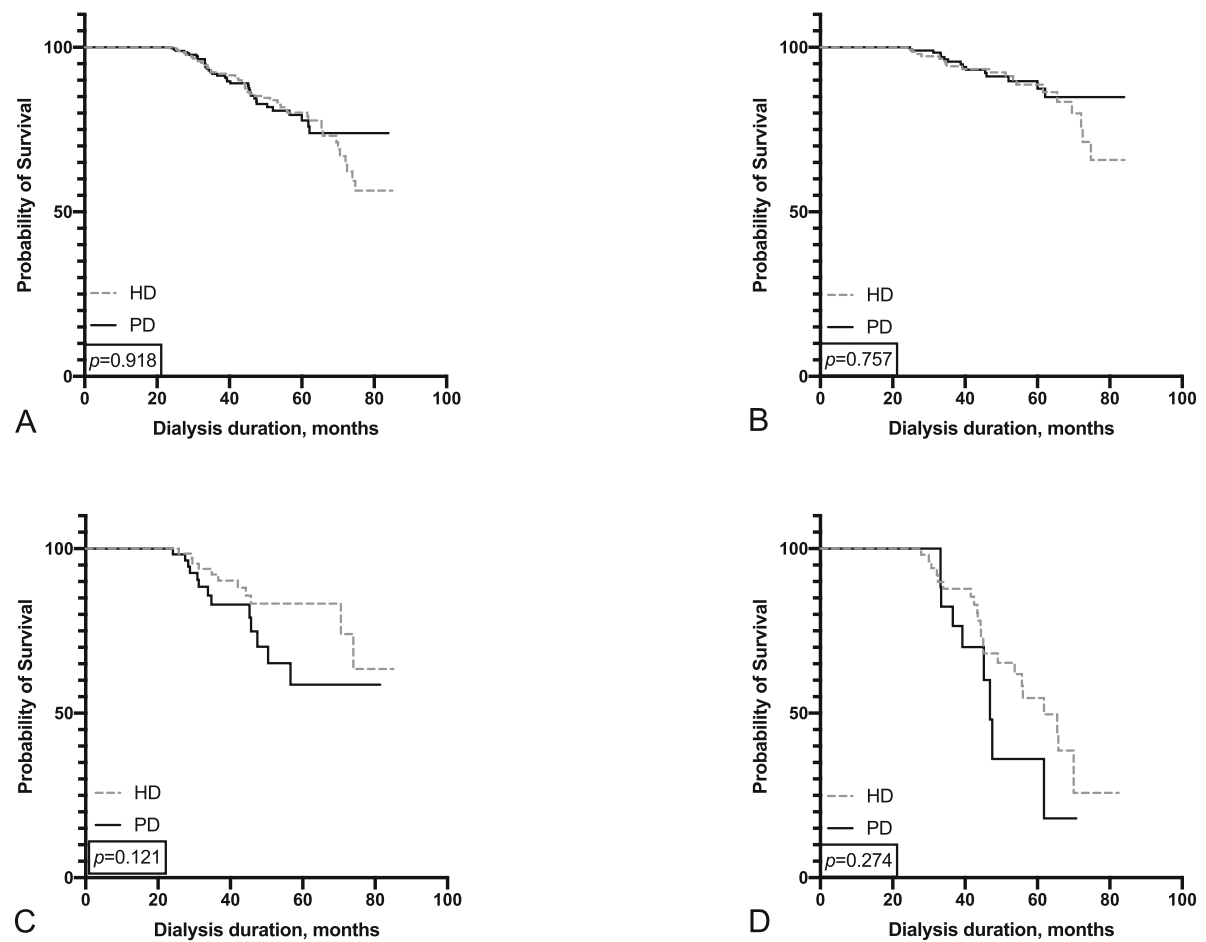

Fig. 4 Comparison of survival between hemodialysis and peritoneal dialysis by age groups from 2 years to end of follow up. (a: all patients, b: $<=$ 60 years old group, c: 60-70 years old group, $\mathbf{d}$ : $>70$ years old group)

reason, we calculated the variance inflation factor of the original model. We found mild multicollinearity that is acceptable. We also tried the model using CCI and the model using comorbidities separately. We found that the survival advantage of PD in the $\leq 60$-year-old subgroup remained. For the $>70$-year-old subgroup, the advantage of HD in the multivariate Cox regression remained, but the advantage in the Cox regression model after PSM disappeared. Generally, it may not be appropriate to use both comorbidities and CCI as covariates in one model, considering that the degree of multicollinearity is acceptable, the results obtained by different models differ little, and we still maintain the original model.

People who are on PD often have a more flexible treatment schedule than people on in-center dialysis. Choosing to do PD instead of in-center hemodialysis may enable patients to keep their regular schedule for work, school or other activities. Younger ESRD patients took advantage of this freedom to return to work and thus received better financial support, which in turn prolonged their survival. Older ESRD patients had poorer self-care ability and needed to rely on the assistance of family members and medical institutions. The in-center dialysis provides clinical assessment twice or thrice a week by clinicians and nurses, therefore, it is rather beneficial to older ESRD patients. PD could cause significant protein-energy loss [28], decreased nutritional intake of older patients might be the cause of a shortened life span. Although this research could not prove the above points, it was speculated as a reasonable explanation and a direction for future research.

This study was a single-center study, which had a longer follow-up time and a larger number of cases than other similar studies in our country [13]. For the first time, the survival of PD and HD patients was compared in southern China. Our study provides insight for the selection of dialysis methods for ESRD patients in the city where the research center is located.

\section{Limitations}

The retrospective medical record data might deviate from the judgment of the primary disease of renal failure. Herein, only baseline laboratory test results were recorded, which might alter during dialysis treatment. Therefore, hemoglobin, plasma albumin, calcium, and phosphorus could not reflect the situation of patients in the treatment process. This study does not include information about patient, professional status, education level, and medical insurance, and therefore cannot assess the impact of financial support on patient survival. In addition, no specific cause of death was investigated in this study. 
The high demand for kidney replacement therapy and the shortage of HD equipment have encouraged the clinicians and patients to prefer PD to HD in recent years, which would lead to significant patient selection bias. Our center is located in Guangzhou, one of China's most advanced cities in economy and medical technology, therefore, the population in this study do not represent other regions of China. Above situation may be the reason for the significantly higher overall survival rate of patients in this study. When extrapolating the conclusions of this study, the factors of economic and technical support should be fully considered.

\section{Conclusions}

For younger ESRD patients ( $\leq 60$-year-old), the 1-, 3-, and 5year survival rates of PD were higher than those of HD. For older ESRD patients (> 70-year-old), the 1-, 3-, and 5-year survival rates of PD were lower than those of HD. Therefore, the results suggested that PD may be a better choice for younger ESRD patients, and HD for the older patients.

\section{Abbreviations}

ESRD: End-stage renal disease; HD: Hemodialysis; PD: Peritoneal dialysis; RCT: Randomized controlled trial; CAPD: Continuous ambulatory peritoneal dialysis; ICD: International Classification of Diseases; CCI: Charlson comorbidities index; SD: Standard deviation; PSM: Propensity score matching; HR: Hazard ratio; Cl: Confidence interval

\section{Acknowledgments}

We thank Prof. Cecilia Lundborg (Karolinska Institutet) for critically reading this manuscript. We thank Dr. Gaetano Marrone (Karolinska Institutet) for providing advice on statistical methods. We thank Ruilei Wang, Jiajie Huang, Yuping Zeng, Jianzhi Chen, Haoyang Fu and the department of information of Guangdong Provincial Hospital of Chinese Medicine for providing support on computer technology.

\section{Authors' contributions}

Design of the study: $\mathrm{ZH}, \mathrm{HH}, \mathrm{DFZ}, \mathrm{FL}, \mathrm{XL}$, and $\mathrm{LW}$; data acquisition, creation of patient follow-up registration software, and analysis: JH, YG, JXL, FY, JJL, $Z H, H L, H H, D X Z, Y Z$, and $Y P$; result interpretation: YM, LZ, LL, GS, ZH, DFZ, FL, $\mathrm{XL}$, and $\mathrm{LW}$. The authors drafted and revised the manuscript and approved the final version.

\section{Funding}

This work was supported by the Traditional Chinese Medicine Bureau of Guangdong Province (grant number: 20201162), Construction of National Specialized Diagnosis and Treatment Center of Regional Traditional Chinese Medicine (grant number: 0103020802) and Pilot Construction of Clinical Collaboration between Chinese and Western Medicine in Major and Refractory Disease (grant number: 0103020603).

\section{Availability of data and materials}

The datasets used and/or analyzed during the current study are available from the corresponding author on reasonable request and with permission of Guangdong Provincial Hospital of Chinese Medicine.

\section{Ethics approval and consent to participate}

This study was conducted in accordance with the ethical principles of the Helsinki Declaration, and approved by the Ethics Committee of the Second Affiliated Hospital of Guangzhou University of Chinese Medicine. With the approval of the Ethics Committee of the Guangdong Provincial Hospital of Traditional Chinese Medicine, the data used in this study were exempted from patient consent.
Consent for publication

Not Applicable.

\section{Competing interests}

The authors have no conflicts of interest to declare.

\section{Author details}

'Department of Nephrology, The Second Affiliated Hospital of Guangzhou University of Chinese Medicine (Guangdong Provincial Hospital of Chinese Medicine), No. 111 Dade Road, Guangzhou 510120, Guangdong, China. ${ }^{2}$ The Second Clinical College of Guangzhou University of Chinese Medicine, Guangzhou, Guangdong, China. ${ }^{3}$ Clinical Research and Data Center, South China Research Center for Acupuncture and Moxibustion, Medical College of Acu-Moxi and Rehabilitation, Guangzhou University of Chinese Medicine, Guangzhou, Guangdong, China. ${ }^{4}$ The First Affiliated Hospital of Guangzhou University of Chinese Medicine, Guangzhou, Guangdong, China.

Received: 29 March 2020 Accepted: 15 September 2020

Published online: 24 September 2020

\section{References}

1. Yu X, Yang X. Peritoneal dialysis in China: meeting the challenge of chronic kidney failure. Am J Kidney Dis. 2015;65:147-51.

2. Wang F, Yang C, Long J, Zhao X, Tang W, Zhang D, et al. Executive summary for the 2015 annual data report of the China kidney disease network (ck-net). Kidney Int. 2019;95:501-5.

3. Morton RL, Tong A, Howard K, Snelling P, Webster AC. The views of patients and carers in treatment decision making for chronic kidney disease: systematic review and thematic synthesis of qualitative studies. BMJ. 2010; 340:c112.

4. Korevaar JC, Feith GW, Dekker FW, van Manen JG, Boeschoten EW, Bossuyt PM, et al. Effect of starting with hemodialysis compared with peritoneal dialysis in patients new on dialysis treatment: a randomized controlled trial. Kidney Int. 2003;64:2222-8.

5. Waldum-Grevbo B, Leivestad T, Reisaeter AV, Os I. Impact of initial dialysis modality on mortality: a propensity-matched study. BMC Nephrol. 2015;16: 179.

6. Heaf JG, Wehberg S. Relative survival of peritoneal dialysis and haemodialysis patients: effect of cohort and mode of dialysis initiation. PLoS One. 2014;9:e90119.

7. Kim H, Kim KH, Park K, Kang SW, Yoo TH, Ahn SV, et al. A population-based approach indicates an overall higher patient mortality with peritoneal dialysis compared to hemodialysis in Korea. Kidney Int. 2014;86:991-1000.

8. Yang F, Khin LW, Lau T, Chua HR, Vathsala A, Lee E, et al. Hemodialysis versus peritoneal dialysis: a comparison of survival outcomes in south-east asian patients with end-stage renal disease. PLoS One. 2015;10:e140195.

9. Wong B, Ravani P, Oliver MJ, Holroyd-Leduc J, Venturato L, Garg AX, et al. Comparison of patient survival between hemodialysis and peritoneal dialysis among patients eligible for both modalities. Am J Kidney Dis. 2018; 71:344-51.

10. Yeates K, Zhu N, Vonesh E, Trpeski L, Blake P, Fenton S. Hemodialysis and peritoneal dialysis are associated with similar outcomes for end-stage renal disease treatment in Canada. Nephrol Dial Transplant. 2012;27:3568-75.

11. Mehrotra R, Chiu YW, Kalantar-Zadeh K, Bargman J, Vonesh E. Similar outcomes with hemodialysis and peritoneal dialysis in patients with endstage renal disease. Arch Intern Med. 2011;171:110-8.

12. Chang YK, Hsu CC, Hwang SJ, Chen PC, Huang CC, Li TC, et al. A comparative assessment of survival between propensity score-matched patients with peritoneal dialysis and hemodialysis in Taiwan. Medicine (Baltimore). 2012;91:144-51.

13. Wu B, Wang M, Gan L, Zhao H. Comparison of patient survival between hemodialysis and peritoneal dialysis in a single chinese center. Int Urol Nephrol. 2014;46:2403-7.

14. Quan H, Sundararajan V, Halfon P, Fong A, Burnand B, Luthi JC, et al. Coding algorithms for defining comorbidities in icd-9-cm and icd-10 administrative data. Med Care. 2005:43:1130-9.

15. Wang IK, Lin CL, Yen TH, Lin SY, Sung FC. Comparison of survival between hemodialysis and peritoneal dialysis patients with end-stage renal disease in the era of icodextrin treatment. Eur J Intern Med. 2018;50:69-74. 
16. Weinhandl ED, Foley RN, Gilbertson DT, Arneson TJ, Snyder JJ, Collins AJ. Propensity-matched mortality comparison of incident hemodialysis and peritoneal dialysis patients. J Am Soc Nephrol. 2010;21:499-506.

17. McDonald SP, Marshall MR, Johnson DW, Polkinghorne KR. Relationship between dialysis modality and mortality. J Am Soc Nephrol. 2009;20:155-63.

18. Vonesh EF, Snyder JJ, Foley RN, Collins AJ. Mortality studies comparing peritoneal dialysis and hemodialysis: what do they tell us?. Kidneylnt Suppl. 2006:(103):S3-S11.

19. Matos JP, Almeida JR, Guinsburg A, Marelli C, Barra AB, Vasconcellos MS, et al. Assessment of a five-year survival on hemodialysis in Brazil: a cohort of 3,082 incident patients. J Bras Nefrol. 2011;33:436-41.

20. Kao TW, Huang JW, Hung KY, Chang YY, Chen PC, Yen CJ, et al. Life expectancy, expected years of life lost and survival of hemodialysis and peritoneal dialysis patients. J Nephrol. 2010;23:677-82.

21. Sanabria M, Muñoz J, Trillos C, et al. Dialysis outcomes in Colombia (DOC) study: a comparison of patient survival on peritoneal dialysis vs hemodialysis in Colombia. Kidney Int Suppl. 2008;(108):S165-S172.

22. Santosh S, Chu C, Mwangi J, Narayan M, Mosman A, Nayak R, et al. Changes in pulmonary artery systolic pressure and right ventricular function in patients with end-stage renal disease on maintenance dialysis. Nephrology (Carlton). 2019;24:74-80,

23. Angkananard T, Janma J, Wannasiri T, Sangthong P, Changsirikulchai S. Associations of left ventricular systolic dysfunction with the factors among thai patients on peritoneal dialysis: a cross-sectional study. BMC Nephrol. 2019;20:257

24. Lin YC, Lin YC, Chen HH, Chen TW, Hsu CC, Peng CC, et al. Different effect of hypercholesterolemia on mortality in hemodialysis patients based on coronary artery disease or myocardial infarction. Lipids Health Dis. 2016;15:211.

25. Wang IK, Lin CL, Sung FC. Lower risk of de novo congestive heart failure in peritoneal dialysis patients compared with hemodialysis patients. Int J Cardiol. 2017;229:123.

26. Charlson ME, Charlson RE, Peterson JC, Marinopoulos SS, Briggs WM, Hollenberg JP. The charlson comorbidity index is adapted to predict costs of chronic disease in primary care patients. J Clin Epidemiol. 2008;61:1234-40.

27. Hemmelgarn BR, Manns BJ, Quan H, Ghali WA. Adapting the Charlson comorbidity index for use in patients with ESRD. Am J Kidney Dis. 2003; 42(1):125-32.

28. Han SH, Han DS. Nutrition in patients on peritoneal dialysis. Nat Rev Nephrol. 2012;8:163-75.

\section{Publisher's Note}

Springer Nature remains neutral with regard to jurisdictional claims in published maps and institutional affiliations.

Ready to submit your research? Choose BMC and benefit from:

- fast, convenient online submission

- thorough peer review by experienced researchers in your field

- rapid publication on acceptance

- support for research data, including large and complex data types

- gold Open Access which fosters wider collaboration and increased citations

- maximum visibility for your research: over $100 \mathrm{M}$ website views per year

At BMC, research is always in progress.

Learn more biomedcentral.com/submissions 